\title{
Anatomy by whole body dissection: a focus group study of students' learning experience
}

This article was published in the following Dove Press journal:

Advances in Medical Education and Practice

25 August 2015

Number of times this article has been viewed

\author{
Annette Burgess' \\ George Ramsey-Stewart ${ }^{2}$ \\ 'Central Clinical School, Sydney \\ Medical School, The University of \\ Sydney, ${ }^{2}$ Sydney Medical School, The \\ University of Sydney, Sydney, NSW, \\ Australia
}

Correspondence: Annette Burgess Central Clinical School, Sydney Medical School, The University of Sydney, Building 63, Level 4, Royal Prince Alfred Hospital, Missenden Road, Camperdown, NSW 2050, Australia

Tel +6I 295158172

Email annette.burgess@sydney.edu.au
Background: The social construction of knowledge within medical education is essential for learning. Students' interactions within groups and associated learning artifacts can meaningfully impact learning. Situated cognition theory poses that knowledge, thinking, and learning are located in experience. In recent years, there has been a reported decline in time spent on anatomy by whole body dissection (AWBD) within medical programs. However, teaching by surgeons in AWBD provides unique opportunities for students, promoting a deeper engagement in learning. In this study, we apply situated cognition theory as a conceptual framework to explore students' perceptions of their learning experience within the 2014 iteration of an 8-week elective AWBD course.

Methods: At the end of the course, all students $(n=24)$ were invited to attend one of three focus groups. Framework analysis was used to code and categorize data into themes.

Results: In total, 20/24 (83\%) students participated in focus groups. Utilizing situated cognition theory as a conceptual framework, we illustrate students' learning experiences within the AWBD course. Students highlighted opportunities to create and reinforce their own knowledge through active participation in authentic dissection tasks; guidance and clinical context provided by surgeons as supervisors; and the provision of an inclusive learning community.

Conclusion: Situated cognition theory offers a valuable lens through which to view students' learning experience in the anatomy dissection course. By doing so, the importance of providing clinical relevance to medical teaching is highlighted. Additionally, the value of having surgeons teach AWBD and the experience they share is illustrated. The team learning course design, with varying teaching methods and frequent assessments, prompting student-student and studentteacher interaction, was also beneficial for student learning.

Keywords: anatomy by whole body dissection, surgery, medical program

\section{Background}

Educational theory has made a move away from information processing theory, where socially constructed knowledge is not apparent. ${ }^{1}$ More recently, the notion that increasing student responsibility and participation within a community is essential for learning. ${ }^{2}$ Students' interactions within groups and associated learning artifacts can meaningfully impact learning. Situated cognition theory refers to theoretical frameworks which pose that knowledge, thinking, and learning are located in experience, ${ }^{2}$ and bound to the social, cultural, and physical context. Context is of paramount importance. According to situated cognition theory, students' knowledge is created through learning experiences that: 
1. facilitate knowledge transfer by authentic tasks tied to context;

2. allow memory to be constructed continuously and are context specific;

3. create personal meaning;

4. encourage social interaction among learners, teachers, and the environment.

In recent years, there has been a reported decline in the time spent on anatomy by whole body dissection (AWBD) within medical programs, with obstacles cited such as lack of time and resources, and an increased reliance on technology for teaching. ${ }^{3}$ However, teaching by surgeons in AWBD provides unique opportunities for students, promoting a deeper engagement from students. ${ }^{3}$

Sydney Medical School is now in its 6th year of providing an 8-week optional elective AWBD course for final year medical students. This course is unique within the medical program, and its success in terms of student knowledge acquisition has been widely reported..$^{3-6}$ Most recently, the article published by Sarkis et al. ${ }^{6}$ shows excellent knowledge acquisition and long-term knowledge retention by students in the 2013 AWBD course. The study concludes that surgeon-facilitated anatomical teaching to medical students by whole body dissection significantly improves topographical anatomical knowledge, which is maintained at 8 months post-course.

The purpose of this study was to use situated cognition theory as a conceptual framework to explore students' perceptions of their learning experience within the 2014 iteration of the AWBD course.

\section{Methods}

\section{Participants}

A total of 24 final year medical students participated in a 40-day (272 hours) dissection elective over 8 weeks. Team learning strategies were implemented, ${ }^{4}$ with students allocated in groups of six each to one of four embalmed cadaver subjects. Informed consent was given by the focus group participants.

\section{Supervision}

The course was facilitated by nine highly experienced retired or semi-retired senior surgeons from various surgical specialties, including general surgery, obstetrics, gynecology, cardiothoracic, head and neck surgery, and neurosurgery. These "Supervisors" were present for their area of anatomical expertise. Nine surgical trainees were also recruited as "Demonstrators". At least two surgeons and four demonstrators were present at any one time during the course.

\section{Course design}

Students were required to prepare for class each day by completing set readings from Cunningham's Dissection Manuals. Specific group dissection tasks were clearly outlined on colorcoded spreadsheets for each anatomical region. A typical daily program consisted of a daily dissection briefing given by an allocated student ( 20 minutes); allocated dissection tasks on cadavers in the wet lab ( 3 hours); small group wet prosected specimen demonstrations (1 hour); and a lecture by a senior surgeon regarding the clinical application of the anatomy being dissected (30 minutes). Structured, Clinical, Objective Referenced, Problem-based, Integrated and Organized (SCORPIO) programs were held at least twice weekly, and were facilitated by senior surgeons.

\section{Assessments}

Formal, standardized practice assessments were held precourse, mid-course, end-course and post-course (8 months after the course). Each assessment consisted of four prosected wet specimens. Each student was required to accurately identify five labeled structures on each specimen, with a maximum score of 20 .

\section{Student perception}

At the end of the course, all students were invited to attend one of three focus groups. The focus groups were conducted by the first author, who had no involvement in the administration, teaching, or assessment of the course. The focus group questions were designed to explore students' perceptions of their learning experience during the course. Focus group data were transcribed verbatim. The data were read by the first author and analyzed to identify themes. Situated cognition theory was used to develop a coding framework, which was applied to the full data set. ${ }^{7}$

Ethics approval was obtained from the University of Sydney Human Research Ethics Committee.

\section{Results}

Qualitative data were obtained from a total of three focus groups, in which 20/24 (83\%) students participated. Utilizing situated cognition theory as a conceptual framework, we illustrate the students' learning experiences within the AWBD course.

\section{Learning experiences that facilitate knowledge transfer by authentic tasks tied to context}

Learning is influenced by provision of specific contexts with authentic activities. ${ }^{2}$ As a visceral experience, learning entails 
not just thinking, but also completing authentic tasks that involve the touching and feeling of material. ${ }^{8,9}$

Some students felt that through active participation in dissection tasks, with guidance from experienced surgeons, they could better understand the material:

We can read a textbook and not understand something like the ischiorectal fossa. But until you dissect it out with a surgeon telling you all the key landmarks and the actual way to follow it, it's only then that you understand what they've taught us before in lectures. You just don't get it until you've done dissection guided by someone who knows what they're doing, who dissects in that area for a living.

Students also felt that by gaining practical experience, they would be in a better position in the future to apply what they had learnt:

Just by remembering the clinical relevance of the jugular veins, you can then go, three veins, draining each side of the thyroid because there's another coming out of the top. So the clinical relevance allows you to bring the picture back together of what you're looking for.

I thought the best part of the program was the clinical exposure, like this is how you go about these surgeries that we've been talking about ... like appendectomies.

\section{Learning experiences that allow memory to be constructed continuously and are context specific}

Memory is always "under construction" and material is best learnt within a relevant context. ${ }^{2}$ Throughout the course, an emphasis was placed on the construction of knowledge and knowledge retention through direct active participation.

Students felt that the provision of a clinical context by surgeons aided in their ability to understand and retain information:

Surgeons actually apply a relevance to dissection tasks. If you just look at something in anatomy, it's just in one ear and out the other. But the surgeons actually give you an application. So because you can actually see a relevance for it ... you retain the knowledge a lot better.

Students felt that the surgical experience of the Supervisors allowed them to articulate and explain important information:

We need someone ... supervisors who pick out the relevance ... and teach that to us. The surgeons have a 3D picture in their head. Even if it is a difficult concept for them to explain, they've had to remember it. So they're able to give you ways of remembering that exact thing. Instead of just reading a list out of a book, surgeons could move through like an MRI machine when explaining things to you ...

The surgeons were really adamant about teaching us about how things connect, and I think that creates that whole body map, rather than the distinct parts that don't really join up.

\section{Learning experiences that create personal meaning}

Learning occurs when students create their own unique reality, rather than simply acquiring knowledge. ${ }^{2}$ This is achieved through participation in tasks that are set within a meaningful context. ${ }^{2}$ Students felt that because of the surgeons' surgical experience, they had a unique ability to convey specific anatomical knowledge that is clinically important and relevant to their future work as medical practitioners and surgeons:

The surgeons taught us what is clinically relevant for us. It's probably what's missing in most of our education, that distillation of knowledge. We need fewer resources and to be shown what to learn, as opposed to here's these books or these notes and go learn them. That's not helpful.

Definitely the best part was the clinical experience. That translates - you can learn better when you understand why you need it.

\section{Learning experiences that encourage social interaction among learners, teachers, and the environment}

Learning experiences that promote interactions between learners, teachers, and the environment are key for learning, with meaning being situated in specific contexts. Social interactions in the classroom emphasize the importance of contributions from all learners, teachers, and the environment. ${ }^{2}$ An emphasis on active learner involvement, with information being presented in a number of ways reinforces learning. ${ }^{10,11}$

Learners recognized the impact their personal actions had on shaping the culture of the learning environment. Through the development of culture, a safe environment was created for other learners. ${ }^{8}$ By participating in group activities, and tackling challenging dissection tasks and problems together, students' learning process was enhanced, along with their knowledge acquisition. Opportunities were provided for students to work and learn together as teams, building on each others' knowledge: 
We're in there trying to learn anatomy and helping each other in our teams, and drawing things on the board .... in a group of six we taught each other. We all want to learn and we were teaching each other ... and we all wanted each other to do well in our assessment tasks.

The learning environment that is created must also support the attainment of students' learning outcomes. ${ }^{8}$ Students enjoyed having a mix of formal teaching methods within the course, including SCORPIOs, lectures, and dissection, combined with informal opportunities for learning:

The important thing is that there was formal and informal teaching and assessment and their clinical context. The informal was just as important as the formal. And so we might, for instance, in our cadaver, when we came across a femoral hernia and the professor got everyone around and he showed us just how to excise that femoral hernia and that was just a serendipitous experience that we happened to have. But that opportunity was jumped on. Everyone crowded around and wanted it to be explained again ... that sort of stuff ... that's what makes for a really good learning environment.

The word "cognitive" implies that learning involves not only the skills of the profession, but also the thought process of the experts in the profession, who are familiar with both the culture and the context in which what is learnt will be applied. ${ }^{8}$ Students found that having surgeons teach in their own area of expertise was beneficial to their learning:

It's essential to have surgeons teach us. It makes so much difference when they're talking about their own area. They know it inside out, they know what is common and what is important. They know what's here, what's there, the easy ways to remember it because they've probably taught it to 600 registrars.

Teachers who are experts in the social, cognitive, and tactile aspects of the surgical profession are able to make their reasoning explicit to the learner, and are also central to role modeling. ${ }^{8}$ Being taught by surgeons with relevant experience, made the students feel inspired to learn:

We want to be surgeons so we understand the technique they use and why it is relevant to the anatomy and why this technique is used this way, not to pin any nerve or destroy any structure or how to access it.

Relationship building between the teachers and the learners provides opportunities for teachers to provide clear, constructive, and timely feedback to students, building on strengths, and improving weaknesses. ${ }^{9}$ The students felt that the way they were treated by the Supervisors had a positive impact on their motivation to learn:

The surgeons themselves just treated us as equals who were just starting a journey, a bit further back from when they started it. It was very very flat in terms of hierarchy.

An in-depth knowledge of learner's development levels enables them to increase their capability through increased appropriate supervision and challenges. ${ }^{10}$ Students were motivated to learn because of the Supervisors' commitment to them and their high expectations:

Part of the reason this course works well is because every day you feel motivated. It's very carrot driven rather than stick driven, you want to impress the Supervisors and you want to improve yourself.

\section{Discussion}

By understanding the students' learning experiences, future courses can be improved. ${ }^{12}$ Viewing learning in a classroom through the lens of situation cognition theory provides a useful framework to understand students' insights and perspectives. Situated cognition theory poses that instruction, cognition, and learning occur within complex, social learning environments. ${ }^{2}$

A cornerstone of medical education theory is that knowledge and understanding is more readily developed when explained within the relevant clinical context. ${ }^{13}$ Feedback from participants noted that Supervisors emphasizing the clinical and especially, the surgical applications of the regions being dissected, aided their acquisition of clinical anatomical knowledge. Close attention should be paid to the discussion of real-life situations in relation to context. ${ }^{2}$ Participants commented that the presence of Supervisors, teaching clinical anatomy in their area of expertise and the anatomical information being conveyed within a clinical context, aided their learning.

Certainly, the teacher is one of the most powerful variables in any educational setting. Although subject expertise of the teacher is extremely important in education, equally important is the teacher's actions, attitudes, and enthusiasm for the subject. ${ }^{14,15}$ Students were impressed by the surgeons' expertise and experience in their specialty field, and consequently, their unique ability to convey what is most important and clinically relevant. However, the surgeons' 
encouraging attitude toward students motivated them to learn. The surgeons provided an approach that orientated students to the cultural, social, and humanistic aspects of surgery. ${ }^{16,17}$ The students' commitment to the course was developed through communications and conversations with surgeons. ${ }^{18}$

Situated approaches to teaching that utilize authentic contexts help students to develop a deeper understanding of knowledge that may be more readily retrieved and transferred to various situations. ${ }^{13}$ By carrying out dissection tasks, students found that they were able to develop an understanding of the three-dimensional nature of structures. The dynamics of the learning and teaching process for students was extended by participation from fellow students, surgical registrars, and the senior surgeons. ${ }^{18}$ Working in small groups, students found that they were accountable for their own learning both as individuals and as groups promoting team development. ${ }^{19}$

\section{Limitations}

One limitation of the study is that it is a small-scale study. It is also qualitative in nature and reflects students' perceptions. It should be acknowledged that the students attending the AWBD course chose to do so voluntarily, and therefore, were likely to be enthusiastic about the course.

\section{Conclusion}

Situated cognition theory offered a valuable lens through which to view students' learning experience in the anatomy dissection course. By doing so, the importance of providing clinical relevance to medical teaching was highlighted. Additionally, the value of having surgeons teach AWBD and the experience they share was illustrated. The team learning course design, with varying teaching methods and frequent assessments, which prompted student-student and studentteacher interaction, was beneficial to student learning.

\section{Author contributions}

All authors contributed toward data analysis, drafting and revising the paper and agree to be accountable for all aspects of the work.

\section{Disclosure}

The authors report no conflicts of interest in this work.

\section{References}

1. Kirshner D, Whitson JA, editors. Situated Cognition: Social, Semiotic, and Psychological Perspectives. Mahwah, NJ: Lawrence Erlbaum; 1997.

2. Durning S, Artino A. Situativity theory: a perspective on how participants and the environment can interact: AMEE Guide no 52. Med Teach. 2011;33:188-199.

3. Ramsey-Stewart G, Burgess AW, Hill DA. Back to the future: teaching anatomy by whole-body dissection. Med J Aust. 2010;193:668-671.

4. Burgess AW, Ramsey-Stewart G, May J, Mellis C. Team-based learning methods in teaching topographical anatomy by dissection. ANZ J Surg. 2012;82:457-460.

5. Burgess A, Ramsey-Stewart G, May J, Mellis C. Teaching anatomy by whole body dissection: a community of practice. Int J Clin Skills. 2013;7:6.

6. Sarkis LM, Treble A, Wing LW, Ramsey-Stewart G. Retention of topographical anatomical knowledge following surgeon-facilitated whole-body dissection. ANZ J Surg. 2014;84(11):820-822.

7. Creswell J. Educational Research: Planning, Conducting and Evaluating Quantitative and Qualitative Research. Upper Saddle River, NJ: Merrill; 2002.

8. Schumacher DJ, Englander R, Carraccio C. Developing the master learner: applying learning theory to the learner, the teacher, and the learning environment. Acad Med. 2013;88:11.

9. Rolfe IE, Sanson-Fisher RW. Translating learning principles into practice: a new strategy for learning clinical skills. Med Educ. 2002;36: 345-352.

10. Brydges R, Dubrowski A, Regehr G. A new concept of unsupervised learning: directed self-guided learning in the health professions. Acad Med. 2010;85(10 Suppl):S49-S55.

11. Swanwick T. Informal learning in postgraduate medical education: from cognitivism to 'culturism'. Med Educ. 2005;39:859-865.

12. McMillan W. Moving beyond description: research that helps improve teaching and learning. AJHPE. 2010;2:1.

13. Bransford J, Brown AL, Cocking RR, editors. How People Learn: Brain, Mind, Experience, and School. Washington, DC: National Academy Press; 1999.

14. Spencer J. Learning and teaching in the clinical environment. $B M J$. 2003;326:591.

15. Harden RM, Crosby J. AMEE Guide No 20. The good teacher is more than a lecturer - the twelve roles of the teacher. Med Teach. 2000;22:334-347.

16. Sullivan W. Work and Integrity: In Crisis and Promise of Professionalism in America. 2nd ed. San Francisco: Jossey-Bass; 2005.

17. Sinclair S. Marking Doctors: An Institutional Apprenticeship. Oxford: Berg; 1997.

18. Rogoff B. Apprenticeship in Thinking: Cognitive Development in Social Context. Oxford: Oxford University Press; 1990.

19. Dunaway GA. Adaptation of team learning to an introductory graduate pharmacology course. Teach Learn Med. 2005;17:56-62.
Advances in Medical Education and Practice

\section{Publish your work in this journal}

Advances in Medical Education and Practice is an international, peerreviewed, open access journal that aims to present and publish research on Medical Education covering medical, dental, nursing and allied health care professional education. The journal covers undergraduate education, postgraduate training and continuing medical education

\section{Dovepress}

including emerging trends and innovative models linking education, research, and health care services. The manuscript management system is completely online and includes a very quick and fair peer-review system. Visit http://www.dovepress.com/testimonials.php to read real quotes from published authors.

Submit your manuscript here: http://www.dovepress.com/advances-in-medical-education-and-practice-journal 